\title{
A STOCHASTIC MODEL FOR ESTIMATION OF EXPECTED TIME TO RECRUITMENT WHEN THE THRESHOLD HAS GENERALIZED EXPONENTIATED GAMMA DISTRIBUTION
}

\author{
Manoharan.M \\ Department of community medicine, SRM Medical College and research center, \\ kattangulathur, Chennai-603203 \\ Rajarathinam. A \\ Manonmaniam Sundaranar University, Tirunelveli - 627 012, Tamil Nadu, INDIA \\ *E-mail: manostatmsu@gmail.com
}

\begin{abstract}
In any organization a number of factors are required to generate production activities and the manpower is the most vital factor. The manpower requirements may increase in course of time due to increase in production activities or due to depletion of manpower at random time points. Hence recruitment is to be made whenever the total demands for manpower crosses the so called threshold level. In this paper an estimate of the expected time to recruitment is derived under the assumption that the threshold level is a random variable which follows the Generalized Exponentiated Gamma distribution. The variances also found out. The results are substantiated with numerical examples.
\end{abstract}

Keywords: Expected time $t$, Recruitment, Threshold Level, Breakdown Point and Generalized Exponentiated Gamma Distribution.

\section{Introduction}

There are many organizations in which manpower is one of the important factors for the survival of the organization. A fluctuation in the availability of volume of manpower is quite common. In may be due to increase in the volume of the work scheduled or the depletion of manpower on successive occasions. Hence proper planning of manpower capacity by recruitment is very much essential. Recruitment cannot be at every occasion of fluctuations in manpower requirements or depletion. It is matter of policy that the demand for manpower crosses the random threshold level then recruitment becomes inevitable.

In this paper the expected time to recruitment and its variance is derived using the concept of shock model and cumulative damage process due to Esary et al., (1972). Such models have been developed by Sathiyamoorthi, (1980), Jeeva et.al., (2004) and Kannadasan et.al., (2013). In this paper it is also assumed that the threshold is the random variable which follows Generalized Exponentiated Gamma Distribution (GEGD). The exponentiated gamma (EG) distribution was introduced by Gupta et.al., (1998). 


\section{Assumptions of the Model}

In order to develop the mathematical model, four basic assumptions are made concerning the distributions of the involved random variables. These assumptions are somewhat artificial, but are made because of lack of detailed real-world information on the same.

The organization takes decisions on revising policies at random times, where the interdecision times, which are called epochs, are i.i.d. random variables.

$>$ At every epoch a random number of persons quit the organization, which leads to depletion of man power, available in the organization.

$>$ The organization is exposed to a break down situation when the total man power depletion personnel exceeds a "threshold level".

The threshold level is represented by a random variable which follows the Generalized Exponentiated Gamma Distribution.

\section{Notations}

$X_{i}$ :a continuous random variable denoting the amount of depletion caused to the system due to the exit of persons on the $\mathrm{i}^{\text {th }}$ occasion of policy announcement, $\quad i=$ $1,2,3, \ldots k$ and $X_{i}^{\prime} s$ are i.i.d and $X_{i}=X$ for all $i$. The probability density functions (p.d.f.) is $\mathrm{g}($.) with cumulative density function (c.d.f.) $\mathrm{G}($.).

$Y \quad$ : a continuous random variable denoting the threshold level having the Generalized Exponentiated Gamma Distribution with p.d.f., h(.) and c.d.f., H(.).

$g($.$) : is the p.d.f. of X_{i}$

$g_{k}($.$) : The k- fold convolution of g($.$) i.e., p.d.f. of \sum_{i=1}^{k} X_{i}$

$g^{*}($.$) : Laplace transform of g(.) ; g_{k}^{*}($.$) : Laplace transform of g_{\mathrm{k}}($.

$U$ : a continuous random variable denoting the inter-arrival times between decision epochs, with p.d.f. $\mathrm{f}($.) and c.d.f. $\mathrm{F}($.$) .$

$V_{k}(t): F_{k}(t)-F_{k+1}(t)$

$F_{k}(t) \quad:$ k fold convolution of $\mathrm{F}(\mathrm{t})$.

$S($.$) \quad : The survivor function i.e. P[T>t]=1-S(t)=L(t)$

\section{Model description and solution}

A random variable $X$ has the Generalized Exponentiated Gamma Distribution with $\lambda$ and $\theta$ parameters, if it Cumulative Distribution Function (CDF) is

$$
F(x)=\left[1-e^{-\lambda x}(1+\lambda x)\right]^{\theta} \quad x>0, \theta>0, \lambda>0
$$

Where $\lambda$ and $\theta$ are scale and shape parameters, respectively.

The p.d.f. is

$$
f(x)=\theta \lambda^{2} x e^{-\lambda x}\left(1-e^{-\lambda x}\right)(1+\lambda x)^{\theta-1} \quad x>0, \theta>0, \lambda>0
$$

Now the Survival Function is (SF) in the present model is given as

$\bar{H}(x)=e^{-\lambda x}+\lambda x e^{-\lambda x}-\lambda x$ 
Now, assuming that the threshold Y follows a Generalized Exponentiated Gamma (GEG) distribution with parameter $\lambda$, it can be proved that

$$
\begin{aligned}
& P\left(X_{i}<Y\right)=\int_{0}^{\infty} g_{k}(x)\left(e^{-\lambda x}+\lambda x e^{-\lambda x}\right) d x \\
& =\int_{0}^{\infty} g_{k}(x) e^{-\lambda x} d x+\int_{0}^{\infty} g_{k}(x) \lambda x e^{-\lambda x} d x \\
& =g_{k}^{*}(\lambda)+\lambda g_{k}^{*}(\lambda)
\end{aligned}
$$

The survival function $S(t)$ which is the probability that the system does not fail before time $\mathrm{t}$ is given as

$$
\begin{aligned}
& S(t)=P(T>t)=\sum_{k=0}^{\infty} V_{k}(t) P\left(X_{i}<Y\right)=\sum_{k=0}^{\infty}\left[F_{k}(t)-F_{k+1}(t)\right]\left[g_{k}^{*}(\lambda)+\lambda g_{k}^{*}(\lambda)\right] \\
& =\sum_{k=0}^{\infty}\left[F_{k}(t)-F_{k+1}(t) g_{k}^{*}(\lambda)\right]-\lambda \sum_{k=0}^{\infty}\left[F_{k}(t)-F_{k+1}(t) g_{k}^{*^{\prime}}(\lambda)\right]
\end{aligned}
$$

Where $F_{k}(t)-F_{k+1}(t)$ indicates the probability of exactly $\mathrm{k}$ - occasions in $(0, \mathrm{t})$ by renewal theory

On simplification we get, $\quad=\sum_{k=0}^{\infty}\left[F_{k}(t)-F_{k+1}(t)\right]\left[g^{*}(\lambda)\right]^{k}-\lambda \sum_{k=0}^{\infty}\left[F_{k}(t)-F_{k+1}(t)\right]\left[g^{*^{\prime}}(\lambda)\right]^{k}$

Now $L(t)=1-S(t)$ and hence

$$
L(t)=1-\left\{\sum_{k=0}^{\infty}\left[F_{k}(t)-F_{k+1}(t)\right]\left[g^{*}(\lambda)\right]^{k}-\lambda \sum_{k=0}^{\infty}\left[F_{k}(t)-F_{k+1}(t)\right]\left[g^{*^{\prime}}(\lambda)\right]^{k}\right\}
$$

Taking the Laplace transform of $\mathrm{L}(\mathrm{t})$ we get

$$
l^{*}(s)=1+\lambda+\left[1-g^{*}(\lambda)\right] f^{*}(s) \sum_{k=1}^{\infty}\left[g^{*}(\lambda) f^{*}(s)\right]^{k-1}-\lambda\left[1-g^{*}(\lambda)\right] f^{*}(s) \sum_{k=1}^{\infty}\left[g^{*}(\lambda) f^{*}(s)\right]^{k-1}
$$

Let the random variable $U$ denoting inter arrival time which follows exponential distribution Laplace transforms with parameter $\lambda$. Now, $f^{*}(s)=\left(\frac{c}{c+s}\right)$ substituting in the below equation we get,

$$
l^{*}(s)=1+\lambda+\frac{\left[1-g^{*}(\lambda)\right] f^{*}(s)}{\left[1-g^{*}(\lambda) f^{*}(s)\right]}-\frac{\lambda\left[1-g^{*^{\prime}}(\lambda)\right] f^{*}(s)}{\left[1-g^{*^{\prime}}(\lambda) f^{*}(s)\right]}
$$

On simplification we get,

$$
=1+\lambda+\frac{\left[1-g^{*}(\lambda)\right] c}{\left[c+s-g^{*}(\lambda) c\right]}-\frac{\lambda\left[1-g^{*^{\prime}}(\lambda)\right] c}{\left[c+s-g^{* \prime}(\lambda) c\right]}
$$

$$
\begin{aligned}
E(T) & =-\frac{d}{d s} l^{*}(s) \text { given } s=0 \\
& =\frac{1}{c\left[1-g^{*}(\lambda)\right]}-\frac{\lambda}{c\left[1-g^{*}(\lambda)\right]}
\end{aligned}
$$




$$
\begin{aligned}
& g^{*}(.) \sim \frac{\mu}{\mu+\lambda}, \quad g^{*^{\prime \prime}}(.) \sim-\frac{\mu}{(\mu+\lambda)^{2}} \\
& E(T)=\frac{1}{c\left[1-\frac{\mu}{\mu+\lambda}\right]}-\frac{\lambda}{c\left[1-\frac{\mu}{(\mu+\lambda)^{2}}\right]} \\
& E(T)=\frac{\mu+\lambda}{c \lambda}-\frac{\lambda(\mu+\lambda)^{2}}{c\left[(\mu+\lambda)^{2}+\mu\right]} \\
& E\left(T^{2}\right)=\frac{d^{2}}{d s^{2}} l^{*}(s) \text { Given } s=0
\end{aligned}
$$

Simplification we get,

$$
E\left(T^{2}\right)=\frac{(\mu+\lambda)^{2}}{c^{2} \lambda^{2}}-\frac{\lambda(\mu+\lambda)^{4}}{c^{2}\left[(\mu+\lambda)^{2}+\mu\right]^{2}}
$$

From which Variance can be obtained

$$
\begin{aligned}
& V(T)=E\left(T^{2}\right)-[E(T)]^{2} \\
& =\frac{(\mu+\lambda)^{2}}{c^{2} \lambda^{2}}-\frac{\lambda(\mu+\lambda)^{4}}{c^{2}\left[(\mu+\lambda)^{2}+\mu\right]^{2}}-\left[\frac{\mu+\lambda}{c \lambda}-\frac{\lambda(\mu+\lambda)^{2}}{c\left[(\mu+\lambda)^{2}+\mu\right]}\right]^{2}
\end{aligned}
$$

On simplification we get,

$$
V(\mathrm{~T})=\frac{2(\mu+\lambda)^{3} \mu(\mu+\lambda+1)}{c^{2} \lambda\left[(\mu+\lambda)^{2}+\mu\right]^{2}}
$$

\section{Numerical Illustration}

The mean and variance of time to recruitment is numerically illustrated by varying one parameter and keeping other parameters fixed. The effect of the parameters $\lambda, \mu$ and $c$ on the performance measures is shown in the following table.

\begin{tabular}{|c|c|c|c|c|}
\hline $\mathbf{c = 1}$ & $\boldsymbol{\mu = 0 . 5}$ & $\boldsymbol{\mu}=\mathbf{1}$ & $\boldsymbol{\mu}=\mathbf{1 . 5}$ & $\boldsymbol{\mu}=\mathbf{1 . 5}$ \\
\hline 1 & 1.409 & 2.235 & 3.052 & 3.87 \\
\hline 2 & 0.704 & 1.118 & 1.526 & 1.935 \\
\hline 3 & 0.47 & 0.745 & 1.017 & 1.29 \\
\hline 4 & 0.352 & 0.112 & 0.763 & 0.968 \\
\hline 5 & 0.282 & 0.447 & 0.61 & 0.774 \\
\hline 6 & 0.235 & 0.057 & 0.509 & 0.645 \\
\hline 7 & 0.201 & 0.319 & 0.436 & 0.553 \\
\hline 8 & 0.176 & 0.279 & 0.382 & 0.484 \\
\hline
\end{tabular}




\begin{tabular}{|c|c|c|c|c|}
\hline 9 & 0.157 & 0.248 & 0.339 & 0.43 \\
\hline 10 & 0.141 & 0.224 & 0.305 & 0.387 \\
\hline
\end{tabular}

Table - 1: The values of $E(t)$ for different values of $\mu$

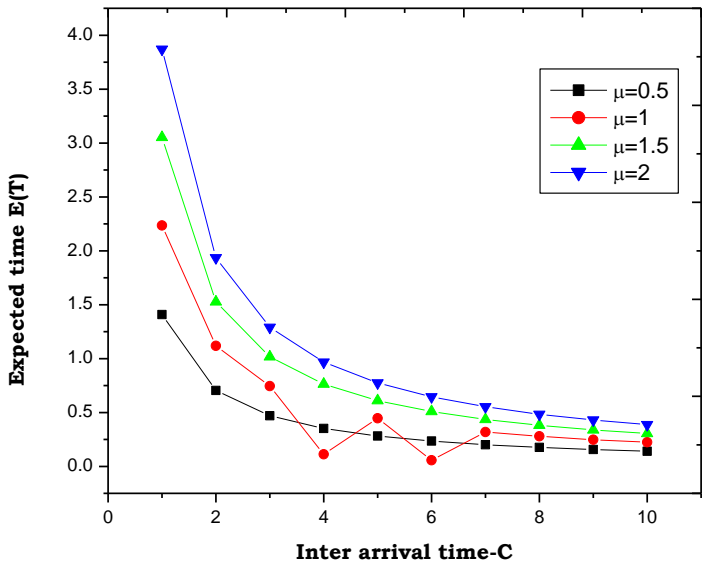

Figure - 1: The values of $E(t)$ for different values of $\mu$

\begin{tabular}{|c|c|c|r|r|}
\hline $\mathbf{c = 1}$ & $\boldsymbol{\mu}=\mathbf{0 . 5}$ & $\boldsymbol{\mu}=\mathbf{1}$ & $\boldsymbol{\mu}=\mathbf{1 . 5}$ & $\boldsymbol{\mu}=\mathbf{2}$ \\
\hline 1 & 1.593 & 2.801 & 4.11 & 5.497 \\
\hline 2 & 0.398 & 0.7 & 1.027 & 1.374 \\
\hline 3 & 0.177 & 0.311 & 0.457 & 0.611 \\
\hline 4 & 0.1 & 0.175 & 0.257 & 0.344 \\
\hline 5 & 0.064 & 0.112 & 0.164 & 0.22 \\
\hline 6 & 0.044 & 0.078 & 0.114 & 0.153 \\
\hline 7 & 0.033 & 0.057 & 0.084 & 0.112 \\
\hline 8 & 0.025 & 0.044 & 0.064 & 0.086 \\
\hline 9 & 0.02 & 0.035 & 0.051 & 0.068 \\
\hline 10 & 0.016 & 0.028 & 0.041 & 0.055 \\
\hline
\end{tabular}

Table - 2: The values of $V(t)$ for different values of $\mu$ are given for in $c$

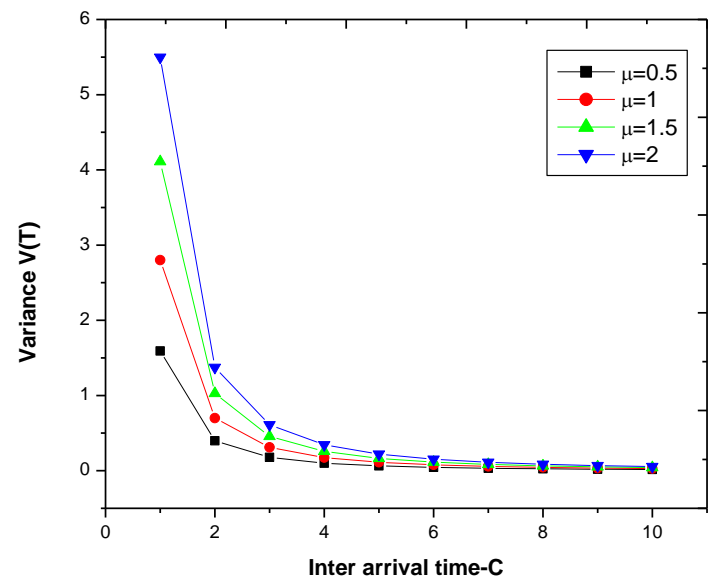

Figure - 2: The values of $V(t)$ for different values of $\mu$ are given for in $c$ 


\begin{tabular}{|c|c|c|c|c|}
\hline $\mathbf{c = 1}$ & $\boldsymbol{\lambda}=\mathbf{0 . 5}$ & $\boldsymbol{\lambda}=\mathbf{1}$ & $\boldsymbol{\lambda}=\mathbf{1 . 5}$ & $\boldsymbol{\lambda}=\mathbf{2}$ \\
\hline 1 & 1.26 & 0.451 & 0.173 & 0.743 \\
\hline 2 & 0.63 & 0.225 & 0.086 & 0.371 \\
\hline 3 & 0.42 & 0.15 & 0.058 & 0.248 \\
\hline 4 & 0.315 & 0.113 & 0.043 & 0.186 \\
\hline 5 & 0.252 & 0.09 & 0.035 & 0.149 \\
\hline 6 & 0.21 & 0.075 & 0.029 & 0.124 \\
\hline 7 & 0.18 & 0.064 & 0.025 & 0.106 \\
\hline 8 & 0.157 & 0.056 & 0.022 & 0.093 \\
\hline 9 & 0.14 & 0.05 & 0.019 & 0.083 \\
\hline 10 & 0.126 & 0.045 & 0.017 & 0.074 \\
\hline
\end{tabular}

Table - 3: The values of $E(t)$ for different values of $\lambda$

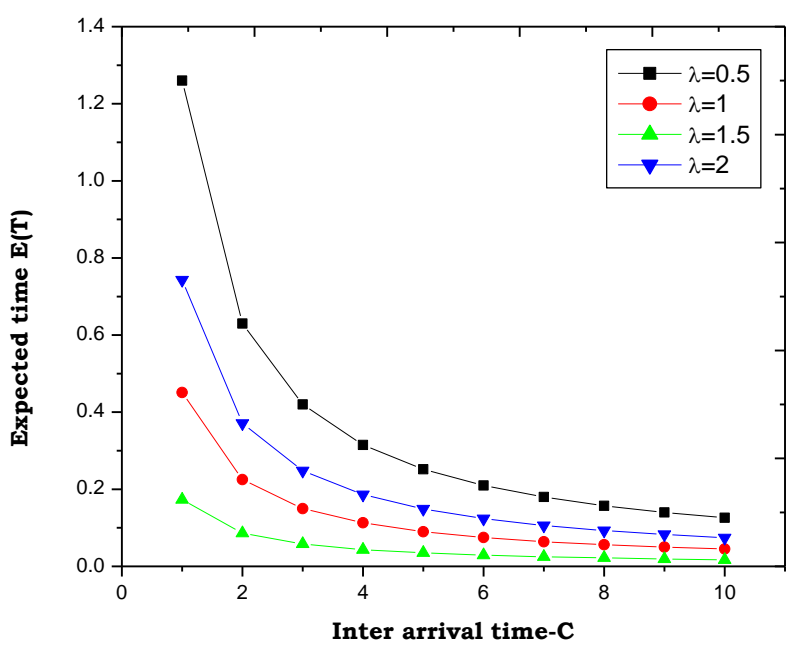

Figure - 3: The values of $E(t)$ for different values of $\lambda$

\begin{tabular}{|c|c|c|c|c|}
\hline $\mathbf{c = 1}$ & $\boldsymbol{\lambda}=\mathbf{0 . 5}$ & $\boldsymbol{\lambda}=\mathbf{1}$ & $\boldsymbol{\lambda}=\mathbf{1 . 5}$ & $\boldsymbol{\lambda}=\mathbf{2}$ \\
\hline 1 & 1.252 & 0.766 & 0.521 & 0.385 \\
\hline 2 & 0.313 & 0.191 & 0.13 & 0.096 \\
\hline 3 & 0.139 & 0.085 & 0.058 & 0.043 \\
\hline 4 & 0.078 & 0.048 & 0.033 & 0.024 \\
\hline 5 & 0.05 & 0.031 & 0.021 & 0.015 \\
\hline 6 & 0.035 & 0.021 & 0.014 & 0.011 \\
\hline 7 & 0.026 & 0.016 & 0.011 & 0.008 \\
\hline 8 & 0.02 & 0.012 & 0.008 & 0.006 \\
\hline 9 & 0.015 & 0.009 & 0.006 & 0.005 \\
\hline 10 & 0.013 & 0.008 & 0.005 & 0.004 \\
\hline
\end{tabular}

Table - 4: The values of $V(t)$ for changes in $\lambda$ 


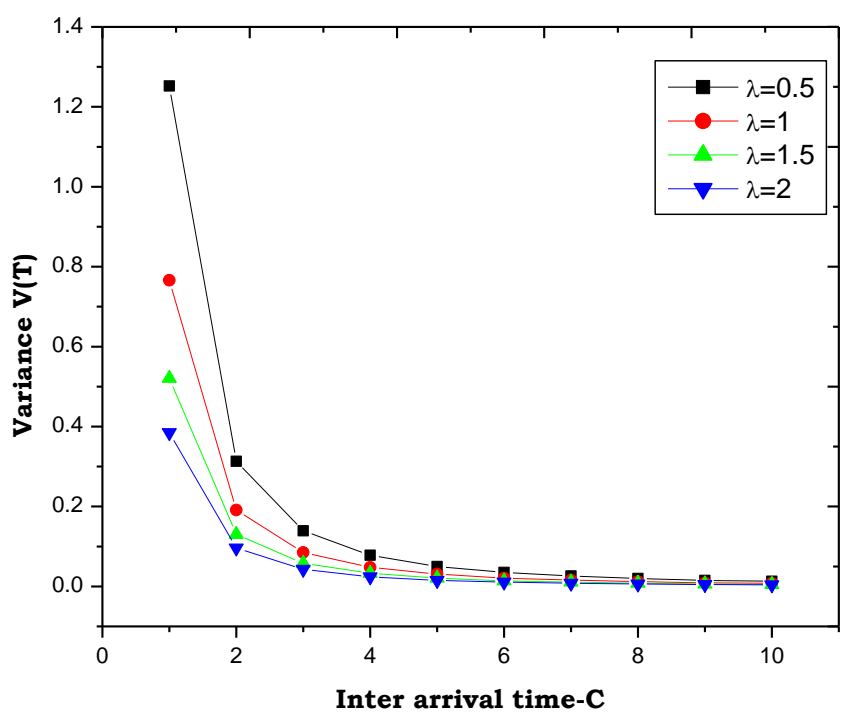

Figure - 4: The values of $\mathrm{V}(\mathrm{t})$ for changes in $\lambda$

\section{Conclusions}

The results revealed that when $\mu$ is kept fixed the inter-arrival time ' $c$ ' which follows exponential distribution, is on the increase and the value of the expected time $E(T)$ to cross the time to recruitment is found to be decreasing, in all the cases of the parameter value $\mu=0.5,1,1.5,2$. When the value of the parameter $\mu$ increases, the expected time is also found decreasing, this is observed in Figure 1. The same case is found in Variance $V(T)$ which is observed in Figure 2.

When $\lambda$ is kept fixed and the parameter of the inter-arrival time ' $c$ ' increases, the value of the expected time $E(T)$ to cross the time to recruitment is found to be decreasing, in all the cases of the parameter value $\lambda=0.5,1,1.5,2$. When the value of the parameter $\lambda$ increases, the expected time is found increasing, this is indicated in Figure 3. The same process is found in the case of V (t) also (Figure 4).

\section{REFERENCES}

1. Barthlomew.D.J.,(1971). The statistical approach to manpower Planning, Statistician, Vol. 20, No.1, pp 3-26.

2. Esary, J.D., Marshall, A.W., and Proschan,F, (1973).Shock models and wear processes, Ann. Probability, Vol.1, No.4, pp.627-649.

3. Ugwuowo, F.I. and Mc Clean,S.I., (2000).Modeling heterogeneity in a manpower system: a review, Applied stochastic models and data analysis, Vol.16, No.2, pp.99-110.

4. Kannadasan, K., Pandiyan, P., Vinoth, V., and Saminathan, R.,(2013).Time to recruitment in an organization through three parameter generalized exponential model, Journal of Reliability and Statistical Studies, Vol.6, No.1, pp. 21-28. 
5. Jeeva,M., Rajalakshmi,R., William, M.L., and Yadavalli, V.S.S. (2004). Expected time for attainment threshold level a shock model approach, Economic Quality Control, Vol 19, No.1, pp.97-105.

6. Gupta,R.C., Gupta, R.D and Gupta,P.L. (1998), Modeling failure time data by Lehman alternatives, Commun. Statist.- Theory Meth., Vol.27(4), pp.887-904.

7. Chandra,S (1990). Attainability of a two characteristics manpower structure, Oper. Research, Vol.27, No. 3, pp. 254-263.

8. Sathiyamoorthi.R. (1980). Cumulative damage model with correlated inter arrival time of shocks, IEEE Transactions on Reliability, R-29, No.3.

9. Seal, H.L. (1945). The Mathematics of a Population Composed of K Stationary Strata, Biometrika, Vol.33, pp.226-230.

10. Shawky, A,I., Bakoban,R, A., (2008).Bayesian and Non-Bayesian Estimations on the Exponentiated Gamma Distribution. Appl. Math. Sci. Vol.51 (2), pp.2521-2530. 